\title{
A Fault Location Method for Multi-terminal Transmission Lines Based on Multiple Current Measurements and Phase Comparison
}

\author{
Zhang Yuanyuan $^{1,}$, Zhu Yongli ${ }^{1, b}$, Zhang Meng ${ }^{1, c}$ and Zhang Ning ${ }^{1, d}$ \\ ${ }^{1}$ School of Electrical \& Electronic Engineering, North China Electric Power University, Baoding \\ 071003, China. \\ azyyxwq0310@163.com, byonglipw@163.com, 'zhangmzwj@163.com, 'zhang_ning26@126.com
}

Keywords: Fault location, multi-terminal transmission lines, phase comparison, multiple current measurements.

\begin{abstract}
Due to the defects of existing fault location methods for multi-terminal transmission lines, such as the fault branches judgement algorithms are too complex to implement, a new fault location method based on phase comparison is presented. In this method, transmission lines are classified into branch and artery section, and the fault intervals and fault phases are determined by comparing the phase between two measurements which installed in the lines using Kaiser self-convolution windows FFT. Based on double-terminal traveling wave theory, the final fault distance can be achieved. Extensive PSCAD/EMTDC simulations are performed to verify the high precision of this method.
\end{abstract}

\section{Introduction}

With the increase of the modern power system capacity, the application of multi-terminal transmission lines in power grid is becoming more and more popular. When a fault occurs, it is significant to find the location as soon as possible. Therefore, researching an accurate fault location method has realistic meaning [1].

Parameter calculation is one of the common used methods for fault location in multi-terminal transmission lines. Because it needs to collect voltage and current at the same time, the calculation process becomes more complex. In recent years, the traveling wave method has been the hot spot in fault location. In literature [2], it first judges one of the branch conditions using two criteria, then judges others using double-terminal traveling wave theory. However, the steps are too complicated to calculate. The literature [3] proposes a fault location scheme based on multiple current points. But these papers are just for two-end transmission lines, rather than multi-terminal lines.

In view of above factors, a novel fault location scheme for multi-terminal transmission lines based on multiple current measurements and phase comparison is proposed in this paper. The fault intervals and fault phases are determined by comparing the phase between two measurements. Based on double-terminal traveling wave theory, the final fault distance can be achieved.

\section{Multiple current measurements phase comparison principle}

The propose of phase comparison principle. The current phase difference principle is applied to fault location by installing current measurements on lines and the measurements are composed of Rogowski current transformers. Rogowski has low price and excellent insulation and it can measure the short circuit current without distortion [4]. Therefore, the current measurements based on current transformers have higher without restriction on installation technology and high cost.

Features of Kaiser window. In the electric power harmonic analysis, the fast Fourier transform (FFT) may produce frequency spectrum leakage and fence effect. Aiming at the flaws of the FFT algorithm, the domestic and foreign experts put forward a variety of algorithms of interpolation of windows FFT. It reduces the spectral leakage error and the interference between harmonics to some degree. 
Kaiser window can customize a set of window function, and the ratio of main lobe and side lobe energy is adjustable. The time domain expression is:

$$
w(n)=\frac{I_{0}\left(\beta \sqrt{1-\left(\frac{n}{N / 2}\right)^{2}}\right)}{I_{0}(\beta)}, 0 \leq|n| \leq N / 2
$$

Where $I_{0}(\beta)$ is the first kind of zero order Bessel function; $\beta$ is the shape parameter; $N$ is the length of the Kaiser window. Kaiser self-convolution window can be obtained after convolution operation of the original function, and the formula for computing as expression (2):

$$
w_{k}^{(m)}(n)=\sum_{x=-\infty}^{+\infty} w_{k}^{(m-1)}(x) w_{k}(n-x)(m \geq 1)
$$

Kaiser self-convolution windowed FFT phase comparison. The operation process of Kaiser self-convolution windowed FFT phase comparison is as follows:

1) Kaiser self-convolution operation and its normalization:

$$
\overline{w_{k}^{(m)}(n)}=\frac{\sum_{-\infty}^{+\infty} w_{k}^{(m-1)}(x) \cdot w_{k}(n-x)}{\sum_{i=0}^{N-1} w_{k}^{(m)}(i)}
$$

2) Add Kaiser self-convolution window for the current fault component $i(n)$ :

$$
i_{w_{k}}(n)=\frac{\sum_{h=0}^{H-1} A_{h} \cos \left(2 \pi h f_{0} n / f_{s}+\varphi_{h}\right) \cdot w_{k}^{(m)}(n)}{\sum_{i=0}^{N-1} w_{k}^{(m)}(i)}
$$

3) Obtain the phase of $i_{\mathrm{wk}}(n)$, then FFT transform, get the real part $\operatorname{Re} I_{w k}(n)$ and the imaginary part $\operatorname{Im} I_{\mathrm{wk}}(k)$ :

$$
\begin{gathered}
\operatorname{Re} I_{w_{k}}(k)=\sum_{n=0}^{N-1} \frac{\sum_{h=0}^{H-1} A_{h} \cos \left(W_{N}^{I_{h}{ }^{n}}+\varphi_{h}\right) w_{k}^{(m)}(n)}{i=\sum_{i=0}^{N-1} w_{k}^{(m)}(i)} \cos W_{N}^{k n} \\
\operatorname{Im} I_{w_{k}}(k)=-\sum_{n=0}^{N-1} \frac{\sum_{h=0}^{N-1} A_{h} \cos \left(W_{N}^{I_{h} n+\varphi_{h}}\right) w_{k}^{(m)}(n)}{\sum_{i=0}^{N-1} w_{k}^{(m)}(i)} \sin W_{N}^{k n}
\end{gathered}
$$

Then the phase response formulas are obtained:

$$
\phi I_{w_{k}}(k)=\arctan \left[\operatorname{Re} I_{w_{k}}(k) / \operatorname{Im} I_{w_{k}}(k)\right]
$$

4) Repeat the above steps and get the phase of each measurement. Next, each two adjacent measurements, assuming $\mathrm{x}$ and $\mathrm{y}$, is presupposed. The phase difference can be obtained finally:

$$
\Delta \phi_{x y}(k)=\left|\phi_{x I_{w k}}(k)-\phi_{y I_{w k}}(k)\right|
$$

5) Judging the state of every line according to the phase difference. Ideally, the phase difference should be the whole $0^{\circ}$ or $180^{\circ}$. That means the phase difference of in phase is $0^{\circ}$, reverse phase is $180^{\circ}$. However, some errors inevitably exist in the process of measurement. Therefore, the in phase deviation is set to $\left[0^{\circ}, 5^{\circ}\right]$, reverse phase deviation is set to $\left[0^{\circ}, 20^{\circ}\right]$, based on literature [3].

\section{The algorithm of fault location}

Three-terminal transmission line fault interval judgement. The diagram of Three-terminal transmission line is shown in figure 1. Define the line MN as artery line, TP as branch lines. The 
current measurements $k_{1}$ and $k_{2}$ is installed on each side of the bus of artery line respectively. The current measurements $k_{3}$ and $k_{4}$ is installed on each side of the bus of branch line respectively. Criteria are shown in table 1 according to different faulted location.

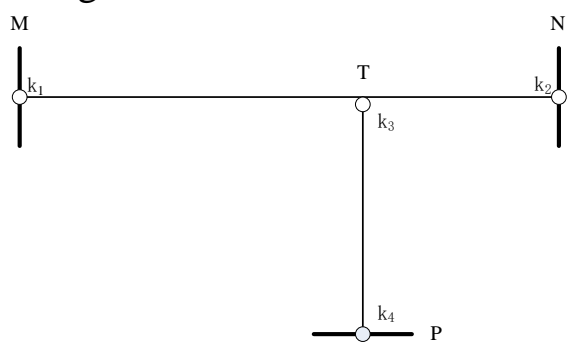

Fig. 1 Three-terminal transmission lines

Tab.1 Fault location criteria of three-terminal transmission lines

\begin{tabular}{ccc}
\hline$\Delta \varphi \mathrm{k} 1 \mathrm{k} 2 /^{\circ}$ & $\Delta \varphi \mathrm{k} 3 \mathrm{k} 4 /^{\circ}$ & Fault interval \\
\hline$[160,180]$ & {$[0,5]$} & $\mathrm{k} 1-\mathrm{k} 2$ \\
{$[160,180]$} & {$[160,180]$} & $\mathrm{k} 3-\mathrm{k} 4$
\end{tabular}

Multi-terminal transmission line fault interval judgement. The diagram of Multi-terminal transmission line is shown in figure 2. Criteria are shown in table 2 according to different faulted location.

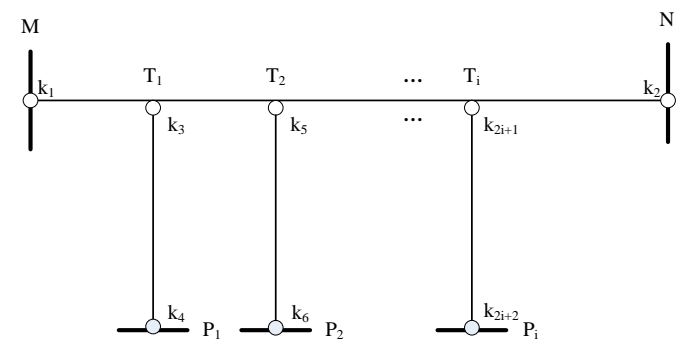

Fig. 2 Multi-terminal transmission lines

Tab.2 Fault location criteria of multi-terminal transmission lines

\begin{tabular}{cccccc}
\hline$\Delta \varphi \mathrm{k} 1 \mathrm{k} 2 /^{\circ}$ & $\Delta \varphi \mathrm{k} 3 \mathrm{k} 4 /^{\circ}$ & $\Delta \varphi \mathrm{k} 5 \mathrm{k} 6 / /^{\circ}$ & $\cdots$ & $\Delta \varphi \mathrm{k} 2 \mathrm{i}+1 \mathrm{k} 2 \mathrm{i}+2 /^{\circ} \cdots$ & Fault interval \\
\hline$[160,180]$ & {$[0,5]$} & {$[0,5]$} & $\cdots$ & {$[0,5]$} & $\mathrm{k} 1-\mathrm{k} 2$ \\
{$[160,180]$} & {$[160,180]$} & {$[0,5]$} & $\cdots$ & {$[0,5]$} & $\mathrm{k} 3-\mathrm{k} 4$ \\
{$[160,180]$} & {$[0,5]$} & {$[160,180]$} & $\cdots$ & {$[0,5]$} & $\mathrm{k} 5-\mathrm{k} 6$ \\
{$[160,180]$} & {$[0,5]$} & {$[0,5]$} & $\cdots$ & {$[160,180]$} & $\mathrm{k} 2 \mathrm{i}+1-\mathrm{k} 2 \mathrm{i}+2$ \\
\hline
\end{tabular}

The fault interval can be identified quickly according to above criteria. Compared with the parameter calculation method, this method does not need to calculate the node voltage through bus voltage data.

\section{Simulation text}

Simulation system and its parameters. For the simulation in PSCAD, the network, depicted in figure 3 . The fault occurs at $0.2 \mathrm{~s}$. The sample rate is $1 \mathrm{MHz}$. The parameters of the lins are shown in table 3.

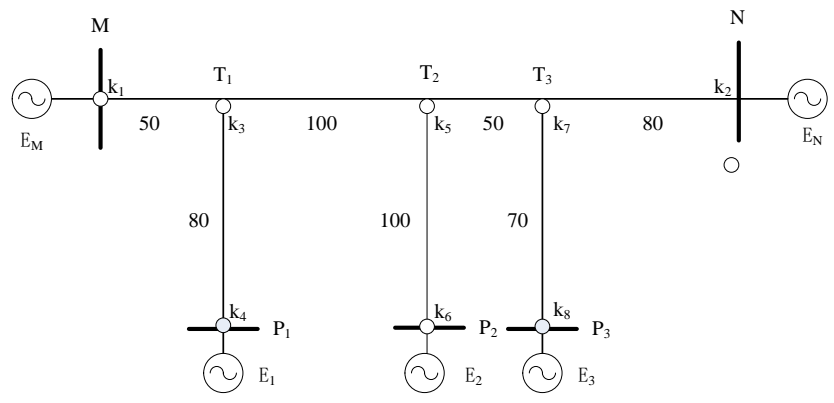

Fig.3 Five-terminal model of the simulated system 
Tab.3 Parameters of transmission lines

\begin{tabular}{ccccc}
\hline Parameter type & $\mathrm{r}(\Omega / \mathrm{km})$ & $\mathrm{x}(\Omega / \mathrm{km})$ & $\mathrm{g}(\mathrm{S} / \mathrm{km})$ & $\mathrm{b}(\mathrm{S} / \mathrm{km})$ \\
\hline Positive sequence & 0.034 & 0.423 & $1 \times 10^{-7}$ & $2.725 \times 10^{-6}$ \\
Negative sequence & 0.300 & 1.142 & $1 \times 10^{-7}$ & $1.935 \times 10^{-6}$ \\
\hline
\end{tabular}

The simulation results and analysis. The fault occurs at $90 \mathrm{~km}$ from $\mathrm{M}$ side with $\mathrm{BC}$ phase to ground fault. The results of phase comparison are shown in table 4.

Tab.4 Results of fault phase and fault interval

\begin{tabular}{cccccc}
\hline Phase comparison & Phase A & Phaes B & Phase C & Fault phase & Fault interval \\
\hline$\Delta \varphi \mathrm{k} 1 \mathrm{k} 2$ & 0.087 & 178.434 & 179.286 & $\mathrm{BC}$ & $\mathrm{k} 1-\mathrm{k} 3$ \\
$\Delta \varphi \mathrm{k} 3 \mathrm{k} 4$ & 0.043 & 7.188 & 2.364 & - & - \\
$\Delta \varphi \mathrm{k} 5 \mathrm{k} 6$ & 2.855 & 0.245 & 2.370 & - & - \\
$\Delta \varphi \mathrm{k} 7 \mathrm{k} 8$ & 1.857 & 0.090 & 1.326 & - & - \\
\hline
\end{tabular}

In table 4, the "- " represents the corresponding phase or interval does not fault. It can be seen that when failure occurs somewhere in the circuit, the application of the proposed method not only can accurately identify the failure interval, it also can identify the fault phase accurately.

Table 5 shows that no matter where the fault occurs, the absolute error is within $1 \%$, which is met with the precision and remedies the defect of traditional fault location methods of the dead zone near the teed node.

Tab.5 Simulation results of fault location

\begin{tabular}{|c|c|c|c|c|c|}
\hline Fault distance & Fault type & $\begin{array}{c}\text { Interval } \\
\text { judgment } \\
\text { result }\end{array}$ & $\begin{array}{l}\text { Phase judgment } \\
\text { result }\end{array}$ & $\begin{array}{l}\text { Measured fault } \\
\text { distance / km }\end{array}$ & $\begin{array}{c}\text { Absolute error / } \\
\mathrm{m}\end{array}$ \\
\hline $\begin{array}{c}\text { MN section from M } \\
\text { 90km }\end{array}$ & BCG & k1-k2 & $\mathrm{BC}$ & 90.094 & 94 \\
\hline $\begin{array}{c}\text { MN section from } \mathrm{N} \\
50 \mathrm{~km}\end{array}$ & AG & k1-k2 & A & 50.157 & 157 \\
\hline $\begin{array}{l}\text { T1-P1 section from P1 } \\
\text { 40km }\end{array}$ & ABCG & k3-k4 & $\mathrm{ABC}$ & 40.088 & 88 \\
\hline $\begin{array}{l}\text { T2-P2 section from P2 } \\
50 \mathrm{~km}\end{array}$ & ABG & k5-k6 & $\mathrm{AB}$ & 50.045 & 45 \\
\hline T3node & CG & k1-k2 & $\mathrm{C}$ & 69.973 & 27 \\
\hline
\end{tabular}

\section{Summary}

This paper proposes a new fault location method for multi-terminal transmission lines based on multiple current measurements and phase comparison. From the relevant simulations, wherever the fault occurs, the fault interval or fault phase can be found, and the ranging relative error is within $1 \%$. Therefore the method proposed in this paper is effective and reliable.

\section{References}

[1] Qian Jiaqi, Ye Jiazhuo, et al. A fault-location method for multi-terminal transmission network based on S transmission [J]. Power System Protection and Control, 2014, 23: 82-88.

[2] Fan Xinqiao, Zhu Yongli, et al. A novel fault location scheme for multi-terminal transmission lines based on principle of double-ended traveling wave [J]. Power System Technology, 2013, 01: 261-269.

[3] Fan Guochen, et al. Fault location scheme for transmission lines using Kaiser self-convolution windowed FFT phase comparison[J]. Power System Protection and Control, 2015, 07: 143-148.

[4] Rigoni M, Garcia J.S.D, Garcia A.P, et al. Rogowski coil current meters [J]. IEEE Potentials, 2008, 27(4): 40-45. 